Journal of Nepal Mathematical Society (JNMS), Vol. 3, Issue 1 (2020); L.de O.Miranda, L.B.B.Miranda

\title{
Lohans' Magic Squares and the Gaussian Elimination Method
}

\author{
Lohans de Oliveira Miranda ${ }^{1}$, Lossian Barbosa Bacelar Miranda ${ }^{2}$ \\ ${ }^{1}$ Unisul University, Santa Catarina, Brazil \\ ${ }^{2}$ IFPI, Piauí, Brazil \\ Correspondence to: Lossian Barbosa Bacelar Miranda, Email: lossianm@gmail.com
}

\begin{abstract}
We have established a new general method to build doubly even magic squares. New types of magic squares are built. The method is aesthetic and easy to understand.
\end{abstract}

Keywords: Arithmetic progressions, Doubly even magic squares, D“urer's magic square, Gaussian elimination method, Parity

DOI: https://doi.org/10.3126/jnms.v3i1.33001

\section{Introduction}

Jacques Sesiano's studies report that the general methods of constructing of doubly even magic squares were made by eastern mathematicians before of the eleventh century ([5], pp. 44-88). Nagarjuna, Indian Buddhist Master who revived the Mahayana, discovered the first non-normal magic square and Narayana says that the magic squares are part of the progressions and that Siva taught them to Manibhadra, the magician (2], p. 51). Nagarjuna's work is the basic source of Buddhism in China and is the synthesis of the previous activities of the Lohans, the great followers of Buddha. Here, we present a new general method which builds, for each order, new types of magic squares hitherto unknown. The ten metric, topological, symmetry and parity properties that these magic squares have make them very useful in art and security. Our method, like that of Nagarjuna exposed in his work Kaksaputa, starts from two arithmetic progressions. Due to this fact, we think that the method is a continuation of the work of the Lohans and we report to them. We use the definitions and notations of [1].

\section{Basic Concepts and Notations Related to the Method}

A magic square of order $n$ is a square matrix formed by the numbers $1,2,3, \cdots, n$ and such that the sum of the numbers of each row, each column and each of the two diagonals is equal to $c_{n}=\frac{n^{3}+n}{2}$. We call $c_{n}$ of magic constant. If $n=4 k, k$ positive natural number, the magic square is of type doubly even magic square.

Let $n=4 u, u \in \mathbb{N}^{*}, I_{n}=\{1,2,3, \cdots, n\}$ and $c_{n}$ the magic constant of $n$ order. We define and denote:

a)

$$
L_{n}=\left(\begin{array}{ccc}
l_{1,1} & \ldots & l_{1, n} \\
\ldots & \ldots & \ldots \\
l_{n, 1} & \ldots & l_{n, n}
\end{array}\right)=\left(l_{u, v}\right)_{u, v \in I_{n}}
$$

the matrix of $n$ order and of $2 \times 2$ blocks determined by

$$
L_{s, r}=\left(\begin{array}{cc}
(n-2(s-1)) n-2(r-1) & (2 s-1) n-(2 r-1) \\
(2 s-1) n+(2 r-1) & (n-2 s) n+2 r
\end{array}\right) ; s, r \in I_{\frac{n}{2}}
$$

b) $H_{n}$, matrix of $n^{\text {th }}$ order generated from of $L_{n}$ by exchanging some entries of the two diagonals with adjacent entries as follows: $l_{1,1}$ with $l_{1,2}, l_{n, 1}$ with $l_{n, 2}, l_{1, n}$ with $l_{1, n-1}, l_{n, n}$ with $l_{n, n-1} ; l_{3,3}$ with $l_{3,4}$, 
$l_{n-2,3}$ with $l_{n-2,4}, l_{3, n-2}$ with $l_{3, n-3}, l_{n-2, n-2}$ with $l_{n-2, n-3}$; etc. Formally, $H_{n}$ is the matrix generated from of $L_{n}$ by the swaps (horizontal swaps): $l_{2 u-1,2 u-1}$ with $l_{2 u-1,2 u} ; l_{n-(2 u-1)+1,2 u-1}$ with $l_{n-(2 u-1)+1,2 u}$; $l_{2 u-1, n-(2 u-1)+1}$ with $l_{2 u-1, n-(2 u-1)} ; l_{n-(2 u-1)+1, n-(2 u-1)+1}$ with $l_{n-(2 u-1)+1, n-(2 u-1)}, u \in I_{\frac{n}{4}}$.

c) $N_{n}=\left(N_{s, r}\right)_{s, r \in I_{n / 2}}$, determined by (inclined swaps)

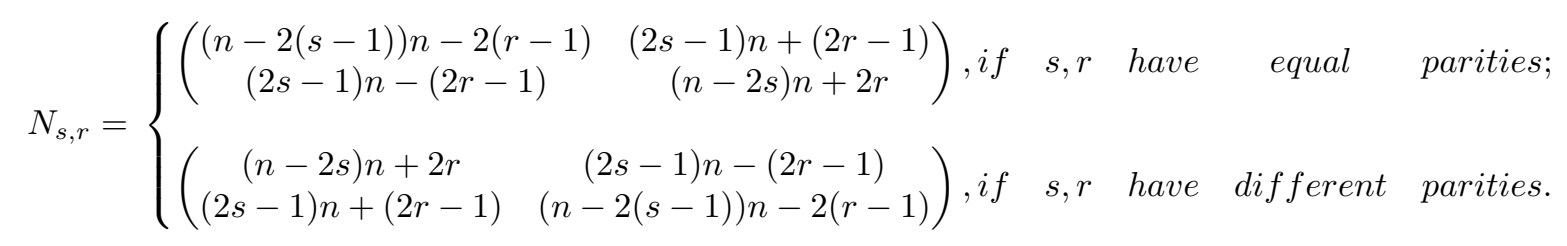

\section{The Main Result}

The proposition below is the essence of the method.

Proposition 1. If in $H_{n}$ we do the same procedure which turns $L_{n}$ into $N_{n}$ (item c) we get a ma$\operatorname{trix} M_{n}$, which is a magic square.

Proof. In $L_{n}$ the sums of the numbers of the first and second rows of the double row of s order are given respectively by

$$
\sum_{r=1}^{n / 2}((n-2(s-1)) n-2(r-1)+(2 s-1) n-(2 r-1))=c_{n}
$$

and

$$
\sum_{r=1}^{n / 2}((2 s-1) n+(2 r-1)+(n-2 s) n+2 r)=c_{n} .
$$

In $N_{n}$ the sums of the numbers of the first and second rows of any double row of odd order s are given respectively by

$$
\begin{array}{r}
\sum_{u=1}^{n / 4}[n-2(s-1)-4(u-1)]+\sum_{u=1}^{n / 4}[(2 s-1) n+4 u-3]+ \\
\quad \sum_{u=1}^{n / 4}[(n-2 s) n+4 u]+\sum_{u=1}^{n / 4}[(2 s-1) n-(4 u-1)]=c_{n}
\end{array}
$$

and

$$
\begin{array}{r}
\sum_{u=1}^{n / 4}[(2 s-1) n-(4 u-3)]+\sum_{u=1}^{n / 4}[(n-2 s) n+4 u-2]+ \\
\sum_{u=1}^{n / 4}[(2 s-1) n+4 u-1]+\sum_{u=1}^{n / 4}[(n-2(s-1)) n-(4 u-2)]=c_{n} .
\end{array}
$$

Similarly, in $N_{n}$, the same result also applies to all double rows of even orders. Using the same procedure as above we also prove that the sums of the numbers of all columns of $N_{n}$ are equal to $c_{n}$. Let $r^{\prime}=\frac{n}{2}-r+1$. So, from (1) results

$$
L_{s, r^{\prime}}=\left(\begin{array}{cc}
(n-2(s-1)) n-(n-2 r) & (2 s-1) n-(n-2 r+1) \\
2 s n-2 r+1 & (n-2 s) n+n-2 r+2
\end{array}\right) .
$$


Note that

$$
\left[(n-2(s-1)) n-2\left(r^{\prime}-1\right)\right]-\left[(n-2 s) n+2 r^{\prime}\right]=-[(2 s-1) n-(2 r-1)]-[(2 s-1) n+(2 r-1)]
$$

and

$$
\left[(2 s-1) n-\left(2 r^{\prime}-1\right)\right]-\left[(2 s-1) n+\left(2 r^{\prime}-1\right)\right]=-[(n-2(s-1)) n-2(r-1)]-[(n-2 s) n+2 r],
$$

indicating that the inclined swaps, when done at $L_{n}$ to generate $N_{n}$, do not change the sum of the numbers of the rows of $L_{n}$, which is $c_{n}$. Horizontal swaps followed by inclined swaps (together) result on: inclined swaps, which cancel each other out (due to the fact that they transfer opposite values to the first row); swap $(2 s-1) n-(n-2 r+1)$ with $(n-2 s) n+n-2 r+2$ and $(n-2(s-1)) n-2(r-1)$ with $(2 s-1) n+(2 r-1)$. These two swaps imply transfers of opposites values to the first row of the double order $s$, whether $r=s$, $r=\frac{n}{2}-s+1$ or any other situation. Therefore, a sum of the numbers of any row of $M_{n}$ is $c_{n}$. Note that the numbers of the first column of the double column of odd order $2(u-1)+1$ of $M_{n}$ are the same as the first column of the double column of $2(u-1)+1$ order of $N_{n}$. In fact, if in (1) we do $s=r$ and establish only inclined swaps results

$$
N_{s, s}=\left(\begin{array}{cc}
(n-2(s-1)) n-2(s-1) & (2 s-1) n+(2 s-1) \\
(2 s-1) n-(2 s-1) & (n-2 s) n+2 s
\end{array}\right) .
$$

If in (1) we do $s=r$ and establish horizontal swaps followed by inclined swaps results

$$
M_{s, s}=\left(\begin{array}{cc}
(2 s-1) n-(2 s-1) & (2 s-1) n+(2 s-1) \\
(n-2(s-1)) n-2(s-1) & (n-2 s) n+2 s
\end{array}\right) .
$$

Then, in the block of $(s, s)$ order there will be only the swaps of positions of the numbers $(n-2(s-1)) n-2(s-$ $1)$ and $(2 s-1) n-(2 s-1)$. Similarly in the double block of order $(n / 2-(2(s-1)+1)+1, n / 2-(2(s-1)+1)+1)$ there will only be swaps of positions between numbers. In the other positions, the numbers of first column of $M_{n}$ and of $N_{n}$ are equal. The proof for the second column is identical. The proof for the columns of even order follows the same reasoning. Then, the sum of the numbers of any column of $M_{n}$ is equal $c_{n}$. Note that the numbers of the main diagonal and of the secondary diagonal remain unchanged by inclined swaps. Therefore, the diagonals of $M_{n}$ and $H_{n}$ are respectively equal. From (1) follows that the sum of numbers of the main diagonal of $L_{n}$ is equal to

$$
\sum_{s=1}^{n / 2}((n-2(s-1)) n-2(s-1)+(n-2 s) n+n-2 s+2)=c_{n}+\frac{n}{2} .
$$

Doing $r=n / 2-s+1$ in (1), we see that the sum of numbers of the secondary diagonal of $L_{n}$ is equal to $c_{n}-n / 2$. On the other hand, the sum of the numbers of the main diagonal of $H_{n}$ is equal to sum of the numbers of the main diagonal of $L_{n}$ minus the sum of the values retired through of the horizontal swaps, namely,

$$
\begin{array}{r}
\left(c_{n}+\frac{n}{2}\right)-\sum_{u=1}^{n / 4}(((n-2(u-1)) n-2(u-1))-((2 u-1) n-(2 u-1)))- \\
\quad \sum_{u=1}^{n / 4}(((2 u-1) n-2(u-1))-((n-2(u-1)) n-(2 u-1)))=c_{n} .
\end{array}
$$

For secondary diagonal of $H_{n}$, the sum of the diferences between the horizontally exchanged numbers is equal to

$\sum_{u=1}^{n / 4}((2(u-1) n+(2 u-1))-(n-(2 u-1) n+2 u))+\sum_{u=1}^{n / 4}(((n-(2 u-1)) n+(2 u-1))-(2(u-1) n+2 u))=-\frac{n}{2}$.

This indicates that after the horizontal swaps the secondary diagonal is left with $n / 2$ units more, therefore the sum of the numbers of the secondary diagonal of $M_{n}$ is $c_{n}$. 


\section{Examples:}

$$
\begin{aligned}
& L_{4}=\left(\begin{array}{cccc}
16 & 3 & 14 & 1 \\
5 & 10 & 7 & 12 \\
8 & 11 & 6 & 9 \\
13 & 2 & 15 & 4
\end{array}\right), H_{4}=\left(\begin{array}{cccc}
3 & 16 & 1 & 14 \\
5 & 10 & 7 & 12 \\
8 & 11 & 6 & 9 \\
2 & 13 & 4 & 15
\end{array}\right) \\
& N_{4}=\left(\begin{array}{cccc}
16 & 5 & 12 & 1 \\
3 & 10 & 7 & 14 \\
2 & 11 & 6 & 15 \\
13 & 8 & 9 & 4
\end{array}\right), M_{4}=\left(\begin{array}{cccc}
3 & 5 & 12 & 14 \\
16 & 10 & 7 & 1 \\
13 & 11 & 6 & 4 \\
2 & 8 & 9 & 15
\end{array}\right) \\
& L_{8}=\left(\begin{array}{cccccccc}
64 & 7 & 62 & 5 & 60 & 3 & 58 & 1 \\
9 & 50 & 11 & 52 & 13 & 54 & 15 & 56 \\
48 & 23 & 46 & 21 & 44 & 19 & 42 & 17 \\
25 & 34 & 27 & 36 & 29 & 38 & 31 & 40 \\
32 & 39 & 30 & 37 & 28 & 35 & 26 & 33 \\
41 & 18 & 43 & 20 & 45 & 22 & 47 & 24 \\
16 & 55 & 14 & 53 & 12 & 51 & 10 & 49 \\
57 & 2 & 59 & 4 & 61 & 6 & 63 & 8
\end{array}\right), \quad M_{8}=\left(\begin{array}{cccccccc}
7 & 9 & 52 & 5 & 60 & 13 & 56 & 58 \\
64 & 50 & 11 & 62 & 3 & 54 & 15 & 1 \\
34 & 23 & 21 & 27 & 38 & 44 & 42 & 31 \\
25 & 48 & 46 & 36 & 29 & 19 & 17 & 40 \\
32 & 41 & 43 & 37 & 28 & 22 & 24 & 33 \\
39 & 18 & 20 & 30 & 35 & 45 & 47 & 26 \\
57 & 55 & 14 & 59 & 6 & 51 & 10 & 8 \\
2 & 16 & 53 & 4 & 61 & 12 & 49 & 63
\end{array}\right) .
\end{aligned}
$$

\section{Some Properties of the Magic Squares}

The magic squares $M_{n}$ (for $n \geqslant 4$ ), which we call magic squares of the Lohans, have the following properties:

i) In each of the rows, columns and diagonals there are exactly $n / 2$ odd numbers. The same goes for even numbers;

ii) The odd numbers are in radial symmetry with respect to the intersection point of the diagonals, as are the even numbers. The difference between two symmetrical odd numbers is a multiple of $n$, and the same is true for even numbers. We have $m_{i, n+1-j}-m_{n+1-i, j}$ is divisible by $\mathrm{n}$;

iii) Both odd and even numbers are in bilateral symmetry with respect to the diagonals and the two orthogonal axes that make an angle of $45^{\circ}$ to them;

iv) The set of odd numbers corresponds to a set connected by paths. The same goes for even numbers;

v) Every odd number is surrounded by even numbers and, also, every even number is surrounded by odd numbers;

vi) For each $n$ the sum of the corners of each of central squares is equal to $S(n)=2\left(n^{2}+1\right)$;

vii) For each $n$,

$$
\begin{aligned}
& \operatorname{Det}\left(\begin{array}{cccc}
n^{2}-2 s n+2 n-2 r+2 & 2 s n-n-2 r+1 & n^{2}-2 s n+2 n-2 r & 2 s n-n-2 r-1 \\
2 s n-n+2 r-1 & n^{2}-2 s n+2 r & 2 s n-n+2 r+1 & n^{2}-2 s n+2 r+2 \\
n^{2}-2 s n-2 r+2 & 2 s n+n-2 r+1 & n^{2}-2 s n-2 r & 2 s n+n-2 r-1 \\
2 s n+n+2 r-1 & n^{2}-2 s n-2 n+2 r & 2 s n+n+2 r+1 & n^{2}-2 s n-2 n+2 r+2
\end{array}\right)=0 \\
& \forall s, r \in I_{n / 2}
\end{aligned}
$$

viii) From (1) we have $m_{i, j}+m_{i, n+1-j}=n^{2}+1, \forall i, j \in I_{n}$; 
Journal of Nepal Mathematical Society (JNMS), Vol. 3, Issue 1 (2020); L.de O.Miranda, L.B.B.Miranda

ix) In the equation (1) the sum of the matrix entries is equal to $2 n^{2}+2$ and in the item vii above the corresponding sum is four times this value;

x) For each $n, \operatorname{Det}\left(M_{n}\right)=0$.

Observation. The item $\mathrm{x}$ is obtained directly by the Gaussian elimination method. The results cited in viii and $\mathrm{x}$ lead us to the following general result on matrices:

Proposition 2. If $M=\left(m_{i j}\right)_{n \times n}$ is a matrix of order $n>2$ and in it the hypothesis $m_{i, j}+m_{i, n+1-j}=$ $k, \forall i, j \in I_{n}(k$ constant $)$ is valid, then $\operatorname{Det}(M)=0$.

Proof. This result is also proved by the Gaussian elimination method. However, for even orders, this proposition can be proved easily using the fact that the determinant is an n-linear function in relation to the columns of the matrix. In particular, we can use it to prove that $\operatorname{Det}\left(M_{n}\right)=0$.

\section{Discussion}

In the method the number 11 is never in the corners as usually happens in the other methods. The method is not a special case of any established methods. For the case $n=4$ the magic square has all the beautiful properties of Dürer's magic square, differing only as follows: in Dürer's magic square we have $9+3+8+14=$ $5+2+12+15=34$ and in the case presented here we have $((13+5+1+9)+(16+12+4+8)) / 2=34$. However, the sum of the common terms is $5+9+8+12=34$. The aforementioned proposition establishes a new general method to build doubly even magic squares. The presented method has an easy analytical treatment and makes strong use of symmetry and parity.

\section{References}

[1] Andrews, W. S., 1908, Magic squares and cubes, open court, Chicago. Available on https://archive.org/details/magicsquarescube00andrrich/page/n6.

[2] Datta, B. and Singh, A. N., 1992, Magic squares in India, Indian Journal of History of Science, 27(1).

[3] De Miranda, L. O. and Miranda, L., 2012, Semi-magic squares from snake-shaped matrices. Available on http://www.slideshare.net/lossian/semimagic-squares-from-snakeshaped matrices.

[4] De Miranda, L. B. P.; Miranda, L. B. B.; Moreira, S. T. S., 2012, Nuevos métodos de construcción de cuadrados semi-mágicos y sus aplicaciones a la enseñanza de matemática computacional y mecánica clásica. Órgano Editor: Educación Cubana, Dirección de Ciencia y Técnica, Ciudad de la Habana, ISBN 978-959-18-0775-5.

[5] Jacques Sesiano, 2019, Magic squares: Their history and construction from ancient times to AD 1600. Springer Nature Switzerland AG 2019. Available on https://doi.org/10.1007/978-3-030-17993-9.

[6] Miranda, L. O., 2016, Generalization of the Ramanujan's Pythagorean identities. 27th Nordic Congress of Mathematicians, Stockholm, Sweden, 16th March 2016, Poster session, Celebrating 100th anniversary of Institut Mittag-Leffler. Available on https://ifpi.academia.edu/LossianMiranda.

[7] Miranda, L. O. and Miranda, L. B., 2015, Law of the lever and the equilibrium of magic hypercubes. International Conference in Number Theory and Physics, IMPA, Rio de Janeiro. Available on https://ifpi.academia.edu/LossianMiranda. 
[8] Miranda, L. O. and Miranda, L. B., 2018, Stability of Ships with Semimagic Rectangles and Parallelepipeds. International Conference on Industrial Mathematics - ICIM-2018 (FOZ2018 PROGRAM, 2018). Available on https://ifpi.academia.edu/LossianMiranda.

[9] Takao Hayashi, 1987, Varahamihira's Pandiagonal Magic Square of the Order Four. HISTORIA MATHEMATICA, 14, 159-166. Available on https://core.ac.uk/download/pdf/82500954.pdf.

[10] World heritage encyclopedia, 2020, Eighteen Luohans, World Library Foundation. 